\title{
Clinical Utility of Enhanced Relative Activity Recovery on Systolic Myocardial Perfusion SPECT: Lessons from PET
}

\author{
Danai Kitkungvan, Pimprapa Vejpongsa, Ketan P. Korrane, Stefano Sdringola, and K. Lance Gould \\ Division of Cardiovascular Medicine, Department of Internal Medicine, University of Texas Health and Science Center at Houston, \\ Houston, Texas
}

SPECT and PET myocardial perfusion images show greater myocardial intensity and homogeneity in systole than diastole because of greater systolic myocardial thickness, less partial volume loss, and enhanced activity recovery. Consequently, conventional myocardial perfusion images obtained from whole cardiac cycles have lower myocardial intensity and greater heterogeneity than systolic images. Considering relative activity distribution on SPECT systolic images may add clinical utility to whole-cycle images and wall motion. Methods: Patients undergoing coronary angiogram within 4 mo after SPECT myocardial perfusion imaging were reviewed. Images were interpreted by 2 masked interpreters using a 17-segment, 5 -point scale to determine summed rest scores (SSS), summed stress scores, and summed difference scores on conventional and systolic images in 603 patients $(55.6 \%$ no coronary artery disease [no-CAD] and $44.4 \% \mathrm{CAD}$ ). Studies were considered normal when the SSS was less than 4 and summed difference score was less than 2. Results: In the no-CAD group, systolic SSS was lower than SSS from conventional images $(2 \pm 2.3$ vs. $3 \pm 2.6, P<0.001)$. In contrast, SSS derived from systolic and conventional images were not different in the obstructive CAD group $(9.1 \pm 7.6$ vs. $9.2 \pm 7.4$, $P=0.559)$. When systolic images were considered, true-negative studies increased from $27.2 \%$ to $43.3 \%(P<0.001)$ whereas false-positive studies decreased from $28.4 \%$ to $12.3 \%(P<0.001)$. True-positive ( $38 \%$ vs. $37.2 \%, P=0.505)$ and false-negative studies (6.5\% vs. $7 \%, P=0.450$ ) were not significantly changed. Diagnostic accuracy increased from $65.2 \%$ to $80.8 \%(P<0.001)$. Conclusion: For gated SPECT myocardial perfusion imaging, when relative activity distribution on systolic images was considered, false-positive studies were reduced and diagnostic accuracy was improved.

Key Words: systolic myocardial perfusion SPECT; ECG gated SPECT perfusion images; myocardial perfusion imaging; SPECT image artifact

J Nucl Med 2015; 56:1882-1888

DOI: 10.2967/jnumed.115.153759

\section{$\mathbf{S}$} coronary artery disease (CAD) is well established and widely used $(1,2)$. However, SPECT MPI is potentially subject to attenuation,

\footnotetext{
Received Jan. 2, 2015; revision accepted Jul. 16, 2015

For correspondence or reprints contact: K. Lance Gould, Weatherhead PET Center for Preventing and Reversing Atherosclerosis, University of Texas Medical School at Houston, 6431 Fannin St., Room MSB 4.256, Houston, TX 77030.

E-mail: K.Lance.Gould@uth.tmc.edu

Published online Aug. 13, 2015.

COPYRIGHT (C) 2015 by the Society of Nuclear Medicine and Molecular Imaging, Inc.
}

partial-volume loss, and imaging artifacts due to contractile motion (3-5). Despite advances in SPECT MPI, imaging artifacts may lead to false-positive studies and difficulty differentiating true perfusion defects from artifacts in some patients (3-5).

Most SPECT MPI studies are now performed using electrocardiographic gating $(6,7)$ that provides significant clinical information on left ventricular (LV) ejection fraction, wall thickening, and regional wall motion to augment SPECT MPI interpretation (8-10). However, routine interpretation of myocardial perfusion images and their summed stress scoring uses conventional whole-cycle images acquired during the entire cardiac cycle not separate systolic or diastolic gated perfusion images (7-11).

Because diastole comprises two thirds of the cardiac cycle, images acquired during diastole might be expected to have more influence on the conventional whole-cycle images than systolic images. However, diastolic images have substantially less myocardial intensity and count density than systolic images (12-15). For electrocardiography (ECG)-gated myocardial perfusion images even by high-resolution PET, systolic images have significantly greater quantitative recovery of absolute myocardial activity due to less partial-volume loss of the thicker systolic LV wall compared with diastolic wall thickness (14-17).

Similar to PET, in gated SPECT MPI, differences between systolic and diastolic quantitative recovery of myocardial activity arise from greater partial-volume loss caused by thinner diastolic LV wall thickness than in systole relative to the limited resolution of SPECT scanners that affects conventional wholecycle perfusion images (12,17-20). Moreover, even in healthy subjects, myocardial perfusion images have regional variability due to varying LV wall thickness caused by papillary muscles, myocardial fiber orientation, and angled imaging planes (21-24). Therefore, the lower diastolic count recovery, compared with systole, can produce or potentiate image inhomogeneity that may appear as perfusion defects in conventional whole-cycle images of healthy subjects that improve or normalize on systolic images $(25,26)$.

Cardiac translational motion in fixed imaging planes of the scanner also causes a complex difference between systolic and diastolic images, as demonstrated in Figure 1. Normally in systole, the heart and aortic root recoil downward and medially with LV ejection and move upward in diastole (27-29). As a consequence, the apex and inferior wall commonly descend into the distal inferior imaging plane in systole and move up out of this plane in diastole $(14,29-$ 31). This translational motion may leave the distal inferior imaging plane with less activity during diastole, thereby creating artifactual defects in conventional whole-cycle images that are not present on systolic images, as documented on prior ECG-gated PET studies (14,29-31). 


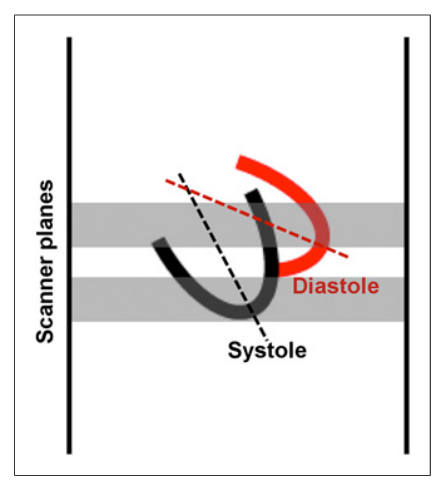

FIGURE 1. Medial and downward recoil of left ventricle during systole may move inferior myocardium into and out of the most inferior imaging plane, thereby reducing inferior activity, especially for inferior apex, with resulting artifact in conventional whole-cycle images not present on systolic images.

LV wall motion on ECGgated perfusion images is commonly used to help differentiate myocardial scar or ischemia from attenuation artifact $(8$ 10). However, our purpose was to test the potential added clinical utility of considering relative activity distribution on systolic images in addition to relative activity and wall motion on standard gated whole-cycle images. Quantitative measurements by ECG-gated PET have proven that systolic perfusion images have quantitatively significantly reduced partial-volume loss and better quantitative activity recovery than diastolic or whole-cycle images (14,29-31). Therefore, for SPECT perfusion imaging, we tested the hypothesis that interpreting relative activity distribution of systolic images would decrease the number of false-positive studies because of reduced partial-volume loss and reduced translational motion on systolic images, thereby improving diagnostic accuracy.

\section{MATERIALS AND METHODS}

\section{Patient Selection}

From January 2010 to December 2013, all sequential patients who underwent stress and rest gated SPECT MPI with ${ }^{99 \mathrm{~m}} \mathrm{Tc}$-sestamibi at Memorial Hermann Hospital, Texas Medical Center, were reviewed. Patients who had a coronary angiogram within 4 mo after gated SPECT MPI were included in the study. The Committees for the Protection of Human Subjects (institutional review board) of the University of Texas and Memorial Hermann Hospital approved the project and determined that signed patient consent for data analysis was not necessary because only deidentified data were used from existing medical records.

Patients with nonischemic cardiomyopathy, previous coronary revascularization, or an interim event were excluded from the study because perfusion defects in these groups may not correlate with the result of coronary angiogram at the time they were performed $(9,32)$. As reported for virtually all MPI-angiogram comparisons in the literature and routine clinical practice, patients were considered to have CAD when the angiogram showed $50 \%$ or greater stenosis of the left main coronary artery or a $70 \%$ or greater stenosis of other epicardial coronary arteries by visual interpretation as entered into the official angiogram report.

\section{Imaging Protocols and Interpretation}

All images were acquired according to the guidelines of the American Society of Nuclear Cardiology (ASNC) using ECG-gated (8 frames per R-R cycle) SPECT single-day or 2-d (depending on body weight) protocols (7). In our institution, only stress images are gated. All $\gamma$ cameras were dual-detector systems, with a $90^{\circ}$ orientation (7). Images were reconstructed primarily by filtered backprojection with a Butterworth prefilter; power of 10; critical frequency of $0.45 \mathrm{Nq}$ for stress, $0.4 \mathrm{Nq}$ for rest images, and $0.35 \mathrm{Nq}$ for ECGgated images at rest and stress; and, less commonly, a quantitative ramp filter or ordered-subset expectation maximization/maximum likelihood expectation maximization, 2 iterations, subset 10 , and the same filters.

Stress was performed by standard exercise treadmill (Bruce or modified Bruce protocol), adenosine infusion (4-min intravenous infusion at a dose of $140 \mathrm{mcg} / \mathrm{kg} / \mathrm{min}$ with or without slow-paced walk on treadmill), regadenoson (single 400 -mcg intravenous bolus), or dobutamine infusion (intravenous infusion at $5-40 \mathrm{mcg} / \mathrm{kg} / \mathrm{min}$ ) (6).

SPECT myocardial perfusion images were interpreted by 2 masked interpreters using the ASNC 17-segment and 5-point qualitative scale ( 0 , normal; 1 , equivocal or mildly reduced; 2 , moderately reduced; 3 , severely reduced radioisotope uptake; and 4 , absence of tracer uptake) to determine a consensus of summed rest score (SRS) and summed stress score (SSS) on conventional whole-cycle images (7). Summed difference score (SDS) is the difference of SSS and SRS indicating reversibility of perfusion defect (7).

In addition, gated stress images were navigated to the single endsystolic frame with the visually smallest LV cavity to calculate systolic SSS and SDS (systolic SSS - SRS). Occasionally for small hearts, the LV cavity nearly disappears as the end systolic frame for analysis. There was no additional training provided to interpreters for systolic image interpretation, who interpreted systolic images as if they were interpreting conventional images. Conventional whole-cycle images and systolic images were considered normal when SSS was less than 4 and SDS was less than 2 (7). LV regional wall motion and thickening were not used for determining SSS and SDS, consistent with ASNC guidelines.

\section{Statistical Analysis}

Demographic and patient characteristics were summarized according to the presence or absence of obstructive CAD using descriptive statistics (mean $\pm \mathrm{SD}$ ) for continuous variables and frequency $(\%)$ for categoric variables. Data were compared using unpaired and paired $t$ tests where indicated for continuous variables. A 2-tailed Fisher exact test and McNemar $\chi^{2}$ test were used to compare paired categoric variables. $P$ values of less than 0.05 indicated statistical significance.

\section{RESULTS}

During the study period, 4,564 sequential patients underwent SPECT MPI at our institution. After inclusion and exclusion criteria were applied, 603 patients meeting criteria were included in our study. The mean age was $57.5 \pm 11.8 \mathrm{y}$, and 366 patients $(60.7 \%)$ were men. Pharmacologic stress with intravenous adenosine infusion was used in 529 patients $(87.7 \%)$, exercise treadmill stress in 52 patients (8.6\%), and regadenoson in 16 patients $(2.7 \%)$. Baseline patient characteristics, risk factors for $\mathrm{CAD}$, and type of stress test are described in Table 1. Significant obstructive CAD by angiography was found in 268 patients (44.4\%). Among patients with CAD (Table 2), single-vessel disease was reported in 147 patients $(54.9 \%)$, and the left anterior descending artery was the most commonly affected (165 patients, $61.6 \%$ ).

Figure 2 shows schematic plots of scanner activity recovery across sections of short-axis images for conventional whole-cycle and systolic images in an example patient with normal coronary artery and no other obvious source of imaging artifact. Because of limited scanner resolution and efficiency at heart depth and associated partial-volume loss, the scanner failed to recover true activity (red dashed line) particularly more so causing relative perfusion defects in anterior and inferior walls in this example. In systole, the LV walls are thicker throughout the heart, with better activity recovery by the scanner and more homogeneous images than the conventional whole-cycle images such that the anterior defect normalizes on systolic images (Fig. 2A). 
TABLE 1

Baseline Patient Characteristics

\begin{tabular}{lccc}
\hline \multicolumn{1}{c}{ Characteristic } & No CAD $(n=335)$ & CAD $(n=268)$ & $P$ \\
\hline Age $(\mathrm{y})$ & $54.8 \pm 11.3$ & $60.9 \pm 11.5$ & $179(66.8)$ \\
Male & $187(55.8)$ & $58(21.6)$ & 0.001 \\
\hline Tobacco use & $46(13.7)$ & $249(92.9)$ & 0.013 \\
\hline Hypertension & $297(88.7)$ & $144(53.7)$ & 0.092 \\
Diabetes & $128(38.2)$ & $188(70.1)$ & $<0.001$ \\
Dyslipidemia & $183(54.6)$ & $28(10.5)$ & \\
Family history of premature CAD & $19(5.7)$ & $240(89.6)$ & 0.001 \\
Type of stress test & & $18(6.7)$ & 0.033 \\
Adenosine & $289(86.3)$ & $7(2.6)$ & 0.261 \\
Exercise & $34(10.2)$ & $3(1.1)$ & 0.147 \\
Regadenoson & $9(2.7)$ & 0.999 & 0.999
\end{tabular}

Values are $n$, with percentage in parentheses, or mean \pm SD. $P<0.05=$ statistically significant.

The inferior defect has similar but less complete activity recovery profiles compared with the anterior defect (Fig. 2B), as expected because of diaphragmatic and liver attenuation. Nonetheless, both these defects substantially improve to nearly normal on systolic images, thereby suggesting that the defects seen in conventional images of this example are artifacts, as confirmed by a normal angiogram.

Examples of conventional whole-cycle and systolic image interpretation are demonstrated in Figure 3. Figure 3A illustrates shortaxis views of an anterior stress perfusion defect on conventional whole-cycle images not present on the systolic images, suggesting that the defect on the whole-cycle image is an artifact, confirmed by coronary angiogram. Figure 3B illustrates an inferior stress defect on both the conventional whole-cycle and the systolic images, suggesting a true defect, confirmed by corresponding stenosis on coronary angiogram.

TABLE 2

Coronary Artery Distribution in Patients with Obstructive CAD

\begin{tabular}{|cc|}
\hline Coronary angiogram & Patient $(n=268)$ \\
\hline CAD & \\
\hline 3-vessel disease & $50(18.7)$ \\
\hline 2-vessel disease & $68(25.4)$ \\
\hline 1-vessel disease & $147(54.9)$ \\
\hline Coronary artery involvement & \\
\hline Left main & $15(5.6)$ \\
\hline Left anterior descending & $165(61.6)$ \\
\hline Left circumflex artery & $117(43.7)$ \\
\hline Right coronary artery & $146(54.5)$ \\
\hline
\end{tabular}

Values are $n$, with percentage in parentheses.

\section{Quantitative Scores of Perfusion Defects}

All calculated SRS, SSS, and SDS were significantly lower in patients with no significant CAD as demonstrated in Table 3. LV ejection fraction in this group of patients was higher than those with $\mathrm{CAD}(61.6 \% \pm 9.8 \%$ vs. $50.6 \% \pm 15.8 \%, P<0.001)$. In patients with no CAD, systolic SSS (Table 4) were significantly lower than SSS calculated from conventional whole-cycle images $(2 \pm 2.3$ vs. $3 \pm 2.6, P<0.001)$. On the other hand (Table 4$)$, the SSS derived from systolic and conventional whole-cycle images were not different in patients with significant CAD $(9.1 \pm 7.6$ vs. $9.2 \pm 7.4, P=0.559$ ). On average (Table 4), SSS calculated from systolic images were lower than those of conventional wholecycle images by $1.0 \pm 1.2$ in patients with no CAD and by $0.1 \pm$ 1.3 in patients with obstructive $\mathrm{CAD}(P<0.001)$.

Integration of systolic images with gated SPECT MPI interpretation (Fig. 4) increased the number of true-negative studies from $164(27.2 \%)$ to $261(43.3 \%, P<0.001)$ whereas false-positive studies decreased from $171(28.4 \%)$ to $74(12.3 \%, P<0.001)$. In patients with obstructive $\mathrm{CAD}$, the number of true-positive and false-negative studies was not significantly changed by adding systolic image interpretation (Fig. 4). Overall, diagnostic accuracy increased from $65.2 \%$ to $80.8 \%(P<0.001)$ when systolic images were incorporated into image analysis (Fig. 4).

The consideration of systolic images did not change interpretation of conventional whole-cycle images (from abnormal to normal or normal to abnormal) in most patients (499 patients, $82.8 \%$ ), as demonstrated in Table 5. For patients with baseline SSS greater than 8 , none of the conventional whole-cycle image interpretations was altered by adding systolic image analysis (Table 5). Among 104 patients (17.2\%) with conventional wholecycle image interpretation altered by adding systolic images, the changed interpretations by considering systolic images correlated with their angiographic data were as follows: false-positive to truenegative in 97 patients (93.3\%), true-positive to false-negative in 5 patients $(4.8 \%)$, and false-negative to true-positive in 2 patients $(1.9 \%)$.

In 97 studies in which interpretation changed from falsepositive to true-negative, 31 patients (32\%) had SSS less than 4 


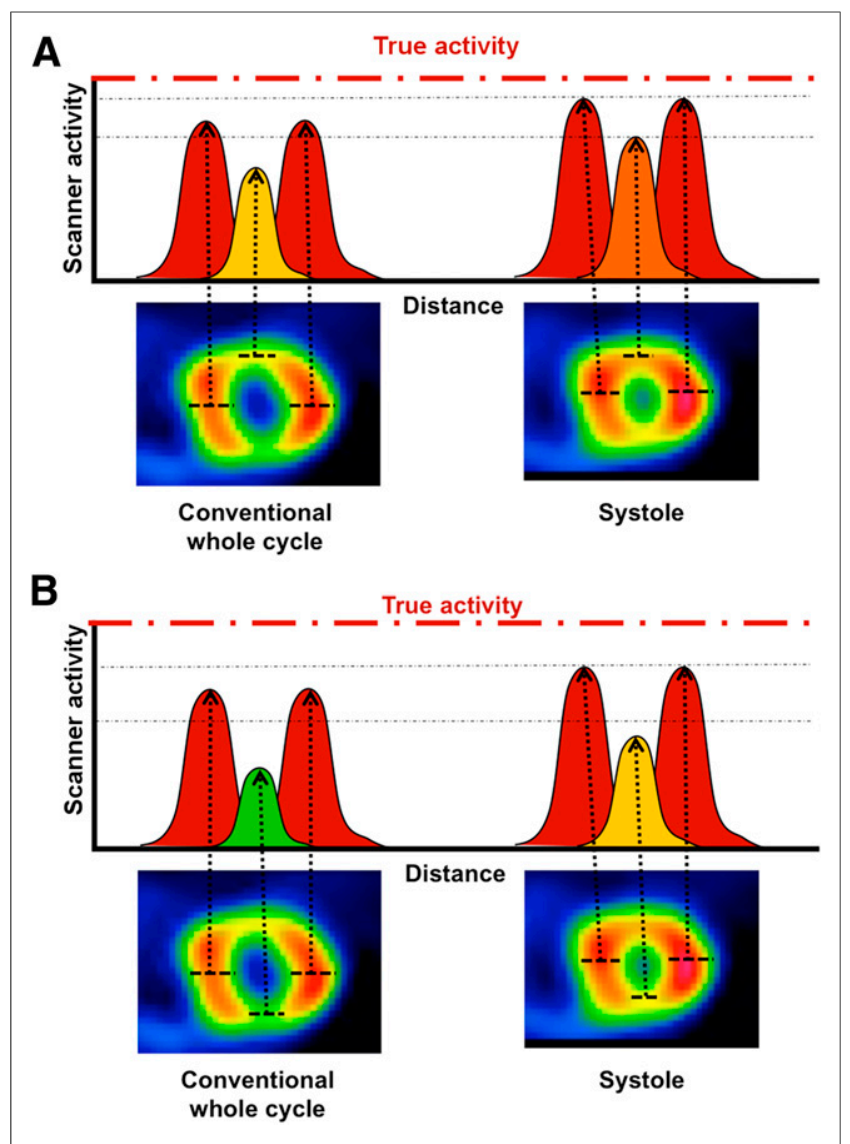

FIGURE 2. In this example with normal angiogram, red dashed line indicates schematically true activity that scanners typically do not recover because of limited resolution and efficiency. Scanner activity recovery in anterior and inferior walls is less on conventional whole-cycle images than systolic images because of limited scanner resolution and partial-volume loss for diastolic images or cardiac motion. This difference between systolic and conventional whole-cycle relative images is less prominent for lateral walls because of their greater thickness due to papillary muscles and associated better activity recovery. (A) In systole, LV walls are thickened with better activity recovery by scanner such that anterior defect normalizes on systolic images, suggesting that defect on conventional image is artifact due to partial-volume loss. (B) Activity recovery profiles for inferior wall during systole compared with anterior defect. This inferior defect also substantially improves to nearly normal on systolic images, suggesting that defect in whole-cycle image is artifact. Some residual attenuation is likely due to diaphragm and liver.

(mean SSS of $2.6 \pm 0.5$ ) but were considered to be abnormal by conventional whole-cycle images due to SDS of 2 or greater (mean SDS of $2.2 \pm 0.4$ ). In 5 studies that changed from truepositive to false-negative, the mean SSS and SDS with conventional method were $3.4 \pm 0.9$ and $1.6 \pm 1.1$, respectively, and $1.8 \pm$ 1.3 and $0 \pm 1.2$, respectively, with added analysis of systolic images.

\section{DISCUSSION}

In our study, perfusion defects on systolic images, as measured by SSS, in patients without obstructive CAD were less severe and smaller than on conventional whole-cycle images because of greater homogeneity and enhanced activity recovery associated with less partial-volume loss and less motion artifact on systolic

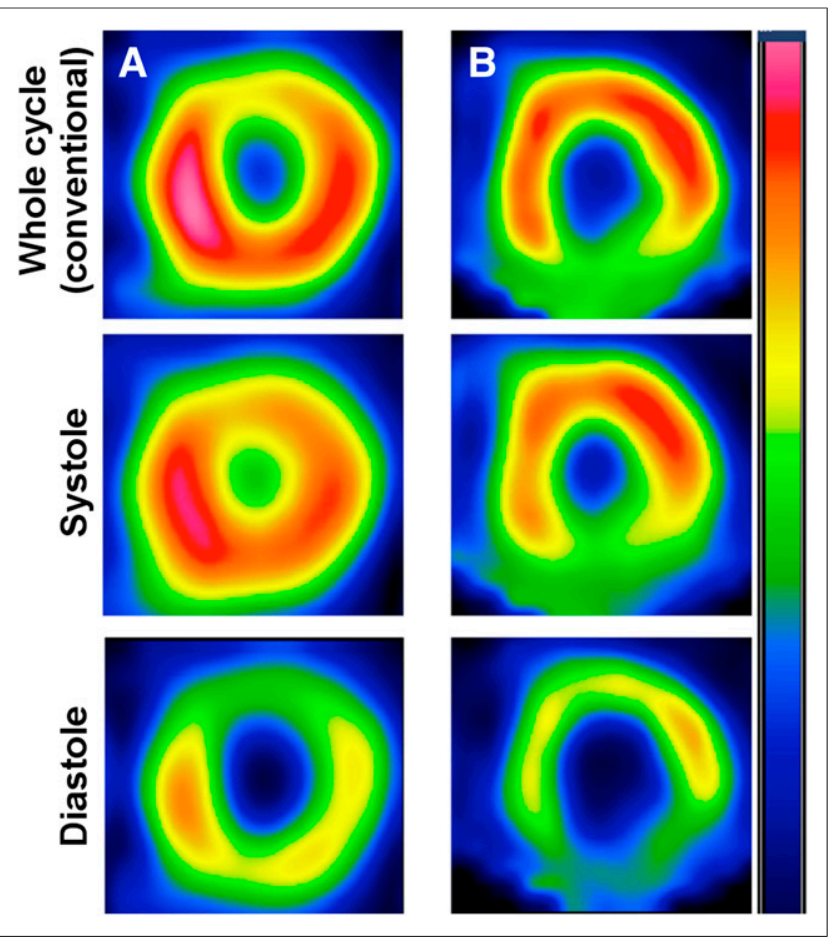

FIGURE 3. (A) Anterior stress perfusion defect on whole-cycle image not present on systolic images suggesting that defect on whole-cycle image is artifact as confirmed by normal coronary angiogram. (B) Inferior stress perfusion defect on both whole-cycle and systolic images indicating true perfusion defect as confirmed by coronary angiogram.

images. By contrast, in patients with obstructive CAD, there was no significant difference in the interpreted size or severity by SSS of perfusion defects between systolic and conventional wholecycle images. These data suggest that artifactual perfusion defects in healthy subjects tend to improve or normalize on systolic images whereas true perfusion abnormalities in patients with obstructive CAD remain unchanged on systolic images. Incorporating systolic images into our routine SPECT MPI analysis

TABLE 3

Interpretation Result by Conventional and Systolic Images in Patients With and Without CAD

\begin{tabular}{|c|c|c|c|}
\hline SPECT score & $\begin{array}{c}\text { No CAD } \\
(n=335)\end{array}$ & $\begin{array}{c}\text { CAD } \\
(n=268)\end{array}$ & $P$ \\
\hline \multicolumn{4}{|l|}{ Conventional images } \\
\hline SRS & $2.2 \pm 2.3$ & $7.6 \pm 7.3$ & $<0.001$ \\
\hline SSS & $3.0 \pm 2.6$ & $9.2 \pm 7.4$ & $<0.001$ \\
\hline SDS & $0.8 \pm 1.7$ & $1.6 \pm 2.6$ & $<0.001$ \\
\hline \multicolumn{4}{|l|}{ Systolic images } \\
\hline Systolic SSS & $2 \pm 2.3$ & $9.1 \pm 7.6$ & $<0.001$ \\
\hline Systolic SDS & $-0.3 \pm 1.8$ & $1.6 \pm 2.8$ & $<0.001$ \\
\hline $\begin{array}{l}\text { Left ventricular } \\
\quad \text { ejection fraction (\%) }\end{array}$ & $61.6 \pm 9.8$ & $50.6 \pm 15.8$ & $<0.001$ \\
\hline $\begin{array}{l}\text { Values are mean } \pm \mathrm{SD} \text {. No } \\
<0.05=\text { statistically signif }\end{array}$ & $\begin{array}{l}\text { rmal study }= \\
\text { ficant. }\end{array}$ & SSS $<4$ and & SDS $<2$ \\
\hline
\end{tabular}


TABLE 4

SSS by Conventional and Systolic Images in Patients With and Without CAD

\begin{tabular}{lccrr}
\hline Patient group & Whole cycle SSS \pm SD & Systolic SSS \pm SD & $P$ & $\Delta S S$ \\
\hline No CAD $(n=335)$ & $3.0 \pm 2.6$ & $2.0 \pm 2.3$ & $<0.001$ & $-1 \pm 1.2^{*}$ \\
CAD $(n=268)$ & $9.2 \pm 7.4$ & $9.1 \pm 7.6$ & 0.559 & $-0.1 \pm 1.3^{*}$
\end{tabular}

Values are mean \pm SD. $\Delta$ SSS $=$ difference between SSS and systolic SSS, normal study $=$ SSS $<4$ and SDS $<2,{ }^{\star} P<0.001$, $P<0.05=$ statistically significant.

improved clinical interpretation by decreasing the number of false-positive studies and increasing true-negative studies and improved overall diagnostic accuracy.

In our study, systolic images altered $32 \%$ of SPECT MPI clinical interpretation from false-positive to true-negative studies based on perfusion defects that normalized on systolic images despite being reversible on conventional rest-stress images. Systole comprises one third of the heart cycle at slow heart rates of resting conditions. At higher heart rates during vasodilator or exercise stress, systole comprises half or more of the cardiac cycle. Accordingly, as previously published in detail for quantitative PET perfusion imaging (14), systolic images have greater impact on whole-cycle images during tachycardia of stress, compared with the slower heart rates at resting conditions. Therefore, SDSs parallel the SSSs.

Systolic image interpretation is most useful and beneficial when perfusion defects are mild because systolic images did not alter interpretation of conventional whole-cycle images when SSS was 8 or greater. Our data indicate that considering systolic images is particularly useful for separating true perfusion abnormalities

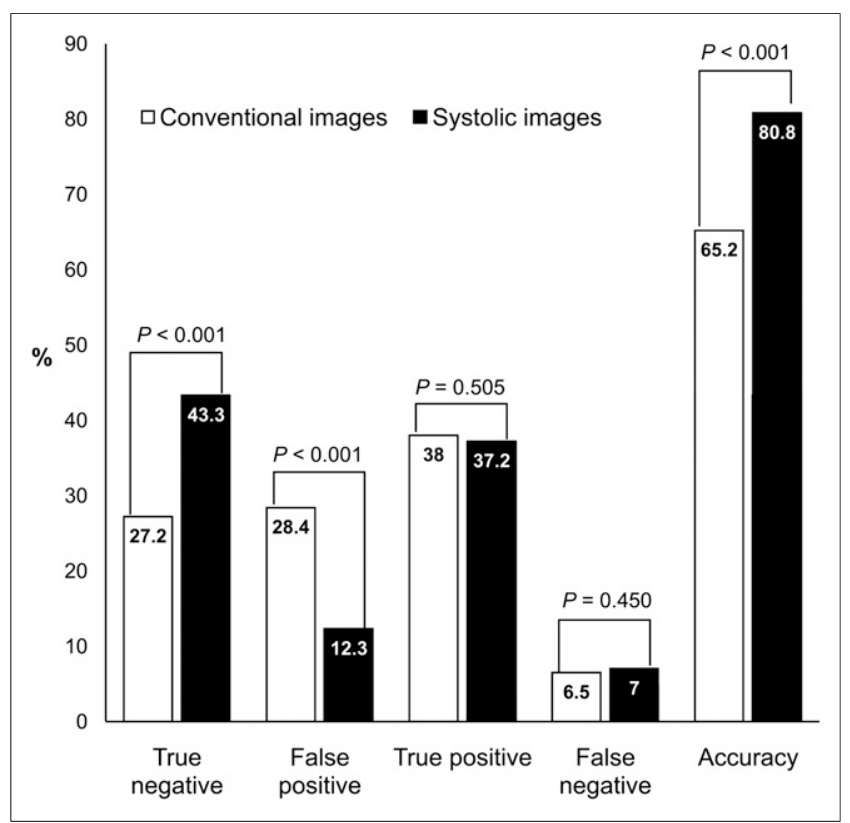

FIGURE 4. Bar graph demonstrates result of considering systolic images in interpretation of whole-cycle conventional images compared with coronary angiogram. from motion artifacts for these mild perfusion defects, as confirmed by coronary angiogram.

Although not statistically significant, in a small number of patients (4.8\% in our study) with small mild stress perfusion defects, systolic image interpretation might cause false-negative results. However, with or without additional information from systolic images, coronary intervention may not be beneficial or indicated in these patients because of small areas of mild myocardial ischemia. With this qualification, the use of systolic images in SPECT MPI interpretation substantially reduced false-positive studies at the small expense of a slight, statistically insignificant increase in number of false-negative studies.

We acknowledge several limitations in our study. Our study is a retrospective, case-control design that cannot account for unmeasured confounders. It was not designed to evaluate sensitivity and specificity of the SPECT MPI compared with coronary angiogram because referral bias could not be objectively considered $(33,34)$. As a university quaternary care center, referral bias or unrecognized confounders likely affected our observed percentage of false-positive and false-negative images but their change by considering systolic images remains valid. Moreover, our observed $28 \%$ false-positive whole-cycle SPECT perfusion images compares with the average of $24 \%$ reported in a large recent metaanalysis with a range of false-positives up to $44 \%-53 \%$ in current practice $(35,36)$.

Although only stress images were ECG-gated for systolic analysis in this study, comparing ECG-gated resting systolic images with stress systolic images would be expected to reduce artifacts of reststress change even more than reported here because the systolic rest images also have thicker LV walls and less partial-volume loss than diastolic or whole-cycle rest images. Whatever small differences in processing between gated and ungated images are unlikely to affect our results because the same gated-ungated processing differences applied to the definite stress defects on systolic and diastolic images confirmed by angiogram were also applied to stress defects that normalized on systolic images in patients with no stenosis on angiogram.

Despite our awareness of and our many publications on its limitations, visual interpretation of coronary stenosis on angiogram was used as a reference standard for binary classification of our study population into significant or nonsignificant coronary artery stenosis, similar to all previous reports on diagnostic accuracy of SPECT MPI in the literature and in widespread clinical practice $(37,38)$.

Although our daily clinical interpretations incorporate wall motion and thickening on gated images, ASNC and American College of Cardiology protocols for qualitative SSS use relative defects without modification of the SSS by wall motion or thickening. 
TABLE 5

Image Interpretation by Conventional and Systolic Images Based on Baseline SSS

\begin{tabular}{|c|c|c|c|c|}
\hline \multirow[b]{2}{*}{ Patient group } & \multirow[b]{2}{*}{ Unchanged* } & \multicolumn{2}{|c|}{ Accuracy } & \multirow[b]{2}{*}{$P$} \\
\hline & & Conventional images & Systolic images & \\
\hline All patients $(n=603)$ & 499 (82.8\%) & $393(65.2 \%)$ & $487(80.8 \%)$ & $<0.001$ \\
\hline SSS $\leq 8(n=481)$ & 377 (78.4\%) & $282(58.6 \%)$ & $376(78.2 \%)$ & $<0.001$ \\
\hline SSS $>8(n=122)$ & $122(100 \%)$ & $111(91 \%)$ & $111(91 \%)$ & - \\
\hline \multicolumn{5}{|c|}{$\begin{array}{l}{ }^{*} \text { Result of interpretation was not altered by systolic images from normal to abnormal or abnormal to normal, normal study }=\text { SSS }<4 \\
\text { and SDS }<2, P \text { value }<0.05=\text { statistically significant. } \\
\text { Values are } n \text {, with percentages in parentheses. }\end{array}$} \\
\hline
\end{tabular}

Moreover, the literature reports no methodology for incorporating wall motion and thickening into SSS of relative perfusion defects.

Separately and in addition to the clinical use of wall motion and thickening, our study addresses the value of considering relative activity distribution on systolic images having enhanced activity uptake with corresponding greater homogeneity and intensity without motion artifacts due to ECG-gated acquisition. Therefore, as similar to all prior reports on SSS in MPI, our study did not incorporate regional LV wall motion and thickening to our wholecycle SPECT MPI SSS (8-10,39,40). Interestingly, considering systolic stress scores provides a potential methodology for semiquantitatively incorporating ECG-gated data into interpretation of relative myocardial perfusion images.

\section{CONCLUSION}

Our study demonstrates that myocardial perfusion defects on conventional whole-cycle SPECT images that visually normalize on systolic images quantified by SSS are artifacts documented by correlation with coronary angiogram. Therefore, integrating relative activity distribution on systolic images combined with interpretation of conventional whole-cycle stress SPECT perfusion images reduces false-positive results with no significant increase in false-negatives and improves overall diagnostic accuracy, thereby potentially reducing the need for diagnostic coronary angiograms.

\section{DISCLOSURE}

The costs of publication of this article were defrayed in part by the payment of page charges. Therefore, and solely to indicate this fact, this article is hereby marked "advertisement" in accordance with 18 USC section 1734. K. Lance Gould receives internal funding from the Weatherhead PET Center for Preventing and Reversing Atherosclerosis endowment. He is also the $510(\mathrm{k})$ applicant for cfrQuant approved by the Food and Drug Administration. He has arranged that all his royalties permanently go to a University of Texas (UT) scholarship fund. UT has a commercial nonexclusive agreement with Positron Corporation to distribute and market cfrQuant in exchange for royalties. However, K. Lance Gould retains the ability to distribute cost-free versions to selected collaborators for research. Additionally, he has signed a nonfinancial, mutual nondisclosure agreement with Volcano Corporation (maker of FFR pressure wires) to discuss coronary physiology projects. No other potential conflict of interest relevant to this article was reported.

\section{REFERENCES}

1. Hendel RC, Berman DS, Di Carli MF, et al. ACCF/ASNC/ACR/AHA/ASE/ SCCT/SCMR/SNM 2009 appropriate use criteria for cardiac radionuclide imaging. J Am Coll Cardiol. 2009;53:2201-2229.

2. Wolk MJ, Bailey SR, Doherty JU, et al. ACCF/AHA/ASE/ASNC/HFSA/HRS/ SCAI/SCCT/SCMR/STS 2013 multimodality appropriate use criteria for the detection and risk assessment of stable ischemic heart disease. J Am Coll Cardiol. 2014;63:380-406.

3. Case JA, Bateman TM. Taking the perfect nuclear image: quality control, acquisition, and processing techniques for cardiac SPECT, PET, and hybrid imaging. J Nucl Cardiol. 2013;20:891-907.

4. Burrell S, MacDonald A. Artifacts and pitfalls in myocardial perfusion imaging. J Nucl Med Technol. 2006;34:193-211.

5. Dvorak RA, Brown RK, Corbett JR. Interpretation of SPECT/CT myocardial perfusion images: common artifacts and quality control techniques. Radiographics. 2011;31:2041-2057.

6. Henzlova MJ, Cerqueira MD, Mahmarian JJ, Yao SS. Stress protocols and tracers. J Nucl Cardiol. 2006;13:e80-e90.

7. Holly TA, Abbott BG, Al-Mallah M, et al. Single photon-emission computed tomography. J Nucl Cardiol. 2010;17:941-973.

8. Smanio PE, Watson DD, Segalla DL, et al. Value of gating of technetium-99m sestamibi single-photon emission computed tomographic imaging. J Am Coll Cardiol. 1997;30:1687-1692.

9. Go V, Bhatt MR, Hendel RC. The diagnostic and prognostic value of ECG-gated SPECT myocardial perfusion imaging. J Nucl Med. 2004;45:912-921.

10. Lima RS, Watson DD, Goode AR, et al. Incremental value of combined perfusion and function over perfusion alone by gated SPECT myocardial perfusion imaging for detection of severe three-vessel coronary artery disease. J Am Coll Cardiol. 2003;42:64-70.

11. Paul AK, Nabi HA. Gated myocardial perfusion SPECT: basic principles, technical aspects, and clinical applications. J Nucl Med Technol. 2004;32:179-187.

12. Fukuchi K, Uehara T, Morozumi T, et al. Quantification of systolic count increase in technetium-99m-MIBI gated myocardial SPECT. J Nucl Med. 1997;38: 1067-1073.

13. Galt JR, Garcia EV, Robbins WL. Effects of myocardial wall thickness on SPECT quantification. IEEE Trans Med Imaging. 1990;9:144-150.

14. Johnson NP, Sdringola S, Gould KL. Partial volume correction incorporating $\mathrm{Rb}-82$ positron range for quantitative myocardial perfusion PET based on systolicdiastolic activity ratios and phantom measurements. J Nucl Cardiol. 2011;18: 247-258.

15. Yamashita K, Tamaki N, Yonekura Y, et al. Quantitative analysis of regional wall motion by gated myocardial positron emission tomography: validation and comparison with left ventriculography. J Nucl Med. 1989;30:1775-1786.

16. Hoffman EJ, Huang SC, Phelps ME. Quantitation in positron emission computed tomography: 1. Effect of object size. J Comput Assist Tomogr. 1979;3:299-308.

17. Bartlett ML, Buvat I, Vaquero JJ, et al. Measurement of myocardial wall thickening from PET/SPECT images: comparison of two methods. J Comput Assist Tomogr. 1996;20:473-481.

18. Pretorius PH, King MA. Diminishing the impact of the partial volume effect in cardiac SPECT perfusion imaging. Med Phys. 2009;36:105-115.

19. Smith WH, Kastner RJ, Calnon DA, et al. Quantitative gated single photon emission computed tomography imaging: a counts-based method for display 
and measurement of regional and global ventricular systolic function. $J$ Nucl Cardiol. 1997;4:451-463.

20. Hutton BF, Osiecki A. Correction of partial volume effects in myocardial SPECT. J Nucl Cardiol. 1998;5:402-413.

21. Pretorius PH, Pan TS, Narayanan MV, King MA. A study of the influence of local variations in myocardial thickness on SPECT perfusion imaging. IEEE Trans Nucl Sci. 2002;49:2304-2308.

22. Bogaert J, Rademakers FE. Regional nonuniformity of normal adult human left ventricle. Am J Physiol Heart Circ Physiol. 2001;280:H610-H620.

23. Ho SY. Anatomy and myoarchitecture of the left ventricular wall in normal and in disease. Eur J Echocardiogr. 2009;10:iii3-iii7.

24. Chareonthaitawee P, Kaufmann PA, Rimoldi O, Camici PG. Heterogeneity of resting and hyperemic myocardial blood flow in healthy humans. Cardiovasc Res. 2001;50:151-161.

25. Bartlett ML, Bacharach SL, Voipio-Pulkki LM, Dilsizian V. Artifactual inhomogeneities in myocardial PET and SPECT scans in normal subjects. J Nucl Med. 1995;36:188-195.

26. Gewirtz H, Tawakol A, Bacharach SL. Heterogeneity of myocardial blood flow and metabolism: review of physiologic principles and implications for radionuclide imaging of the heart. J Nucl Cardiol. 2002;9:534-541.

27. Pratt RC, Parisi AF, Harrington JJ, Sasahara AA. The influence of left ventricular stroke volume on aortic root motion: an echocardiographic study. Circulation. 1976;53:947-953.

28. Beller CJ, Labrosse MR, Thubrikar MJ, Robicsek F. Role of aortic root motion in the pathogenesis of aortic dissection. Circulation. 2004;109:763-769.

29. Loghin C, Sdringola S, Gould KL. Common artifacts in PET myocardial perfusion images due to attenuation-emission misregistration: clinical significance, causes, and solutions. J Nucl Med. 2004;45:1029-1039.

30. Gould KL, Pan T, Loghin C, Johnson N, Guha A, Sdringola S. Frequent diagnostic errors in cardiac PET-CT due to misregistration of CT attenuation and emission PET images: a definitive analysis of causes, consequences and corrections. J Nucl Med. 2007;48:1112-1121.

31. Johnson NP, Pan T, Gould KL. Shifted helical CT to optimize cardiac PET-CT co-registration: quantitative improvement and limitations. Mol Imaging. 2010;9: 256-267.
32. Danias PG, Ahlberg AW, Clark BA 3rd, et al. Combined assessment of myocardial perfusion and left ventricular function with exercise technetium- $99 \mathrm{~m}$ sestamibi gated single-photon emission computed tomography can differentiate between ischemic and nonischemic dilated cardiomyopathy. Am J Cardiol. 1998;82:1253-1258.

33. Ladapo JA, Blecker S, Elashoff MR, et al. Clinical implications of referral bias in the diagnostic performance of exercise testing for coronary artery disease. $\mathrm{J} \mathrm{Am}$ Heart Assoc. 2013;2:e00505.

34. Miller TD, Hodge DO, Christian TF, et al. Effects of adjustment for referral bias on the sensitivity and specificity of single photon emission computed tomography for the diagnosis of coronary artery disease. Am J Med. 2002;112:290-297.

35. Patel MR, Dai D, Hernandez AF, et al. Prevalence and predictors of nonobstructive coronary artery disease identified with coronary angiography in contemporary clinical practice. Am Heart J. 2014;167:846-852.e2.

36. Al Moudi M, Sun Z, Lenzo N. Diagnostic value of SPECT, PET and PET/CT in the diagnosis of coronary artery disease: a systematic review. Biomed Imaging Interv J. 2011;7:e9.

37. Gould KL. Does coronary flow trump coronary anatomy? JACC Cardiovasc Imaging. 2009;2:1009-1023.

38. Parker MW, Iskandar A, Limone B, et al. Diagnostic accuracy of cardiac positron emission tomography versus single photon emission computed tomography for coronary artery disease: a bivariate meta-analysis. Circ Cardiovasc Imaging. 2012;5:700-707.

39. Cerqueira MD, Nguyen P, Staehr P, Underwood SR, Iskandrian AE; ADVANCEMPI Trial Investigators. Effects of age, gender, obesity, and diabetes on the efficacy and safety of the selective A2A agonist regadenoson versus adenosine in myocardial perfusion imaging integrated ADVANCE-MPI trial results. JACC Cardiovasc Imaging. 2008;1:307-316.

40. Hachamovitch R, Hayes SW, Friedman JD, Cohen I, Berman DS. Comparison of the short-term survival benefit associated with revascularization compared with medical therapy in patients with no prior coronary artery disease undergoing stress myocardial perfusion single photon emission computed tomography. Circulation. 2003;107:2900-2907. 